\title{
Synthesis of monodisperse poly(styrene-co-divinyl benzene) microspheres by precipitation polymerization in acetonitrile and $\mathrm{n}$-butanol mixture
}

\author{
Jiang Hongyan, Chen Hou, ${ }^{*}$ Liang Ying, Xuan Yuhong, Wang Mingliang
}

*School of chemistry and Materials Science, ludong University, 264025 Yantai, China; fax:+865356697667; e-mail: sdchenhou@hotmail.com.

(Received: 23 October, 2008; published: 13 September, 2009)

\begin{abstract}
Narrow disperse poly(styrene-co-divinylbenzene) microspheres were prepared by precipitation polymerization using acetonitrile/n-butanol mixture as the reaction medium and 2,2'-azobis(2-methylpropionitrile) (AIBN) as initiator. The resulting particles have smooth surfaces due to the absence of any additional stabilizer. Effects of the reaction parameters such as n-butanol fraction in the reaction medium, monomer and initiator concentration, and divinylbenzene content were investigated. It was found that the particle size increased with an increasing concentration of monomer and initiator, while the uniformity was kept constant. The number-average particle diameter ranged from 2.36 to $1.63 \mu \mathrm{m}$ and decreased with an increasing divinylbenzene concentration. In addition, the uniformity was enhanced as the concentration of divinylbenzene increased. On the basis of the DSC and TGA data, the microspheres prepared by the precipitation polymerization possessed a highly crosslinked structure and the thermal stability improved with the DVB concentration.

Key words: Precipitation polymerization, Microsphere, n-Butanol, Styrene, Divinylbenzene
\end{abstract}

\section{Introduction}

Polymer colloids have been utilized in a wide range of traditional applications such as coating, adhesives, inks, leather finishing, construction, and so forth [1]. Nowadays, such polymer particles have expanded their applications to more advanced fields, including information technology, electric and electronic science, and biotechnology [2-4]. Their applications are dominated by the chemical and physical properties of the polymer. So the precise control of properties of the polymer colloids has become more and more important. Their size, morphology of the polymer beads, uniformity of size, the degree of crosslinking and so forth are the main concerns in controlling properties. In particular, micrometer-sized, highly crosslinked polystyrene (PS) microspheres have received much attention due to the properties such as superior thermal and mechanical resistance, applicability of surface modification, adaptability in a wide $\mathrm{pH}$ range etc [5]. Then the copolymerization of styrene and divinylbenzene system with different polymeric methods has been studied by many researchers [6, 7], such as emulsion polymerization, dispersion polymerization, and suspension polymerization etc. Unfortunately, highly crosslinked and monodisperse polymer microspheres with micron-sized cannot be easily synthesized by any of aforementioned methods. Stöver's group [8] has introduced precipitation polymerization to prepare such particles in single step. Surprisingly, the reactions are carried out in absence of any polymeric stabilizer in an organic medium [9]. To date, several monomers including maleric anhydride [10], methacrylate [11], methyl 
methacrylate [12], chloromethylstyrene [13], glycidyl methacrylate [14], acrylamide [15] and styrene [16] were copolymerized with divinylbenzene as the crosslinkable monomer using precipitation polymerization.

In the precipitation polymerization, acetonitrile meets the general requirement for a precipitation polymerization solvent as it is miscible with monomers and initiator, and is a nonsolvent for the polymer formed [17]. However, other solvents or cosolvents have not been well researched yet. In this study, monodisperse poly(styrene-codivinylbenzene) microspheres were successfully prepared by precipitation polymerization and a new reaction medium was developed based on characteristics such as the morphology, particle size and uniformity, the thermal properties, and the yield of the polymerization. The monodisperse and highly crosslinked microspheres were obtained using the mixture of acetonitrile and $n$-butanol as the polymerization medium in a single step. In addition, low toxicity was one of the advantages for this study.

\section{Results and discussion}

\section{Effect of n-Butanol Concentration on Particle Morphology}

In the precipitation polymerization, acetonitrile meets the solvency conditions required for narrow or monodisperse particle formation. Mixture of acetonitrile with nbutanol also led to monodisperse microspheres.

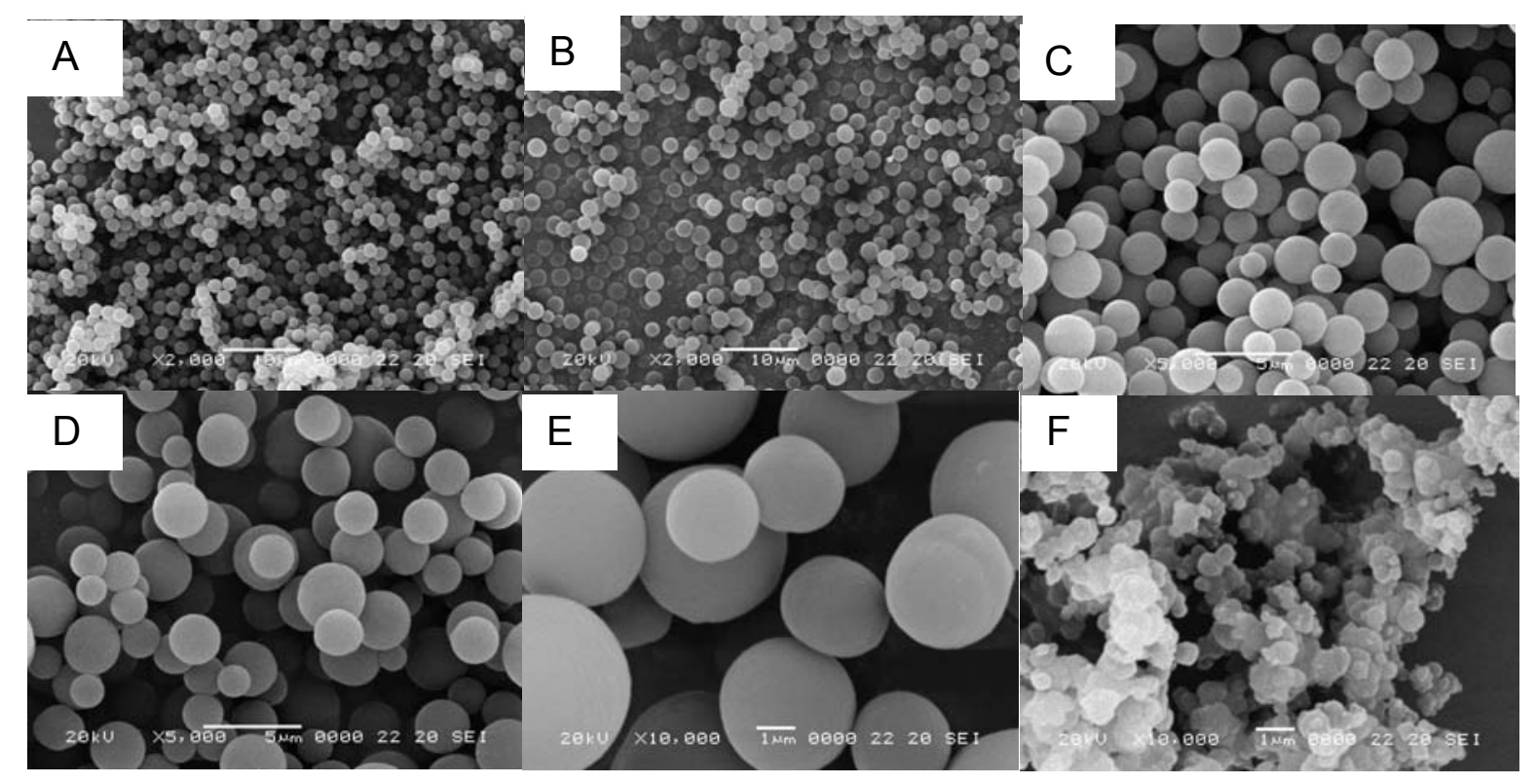

Fig. 1. SEM microphotographs of poly(St-co-DVB) particles prepared by precipitation polymerization composed of various concentration of $n$-butanol with 1 wt. \% AIBN and 1 vol. $\%$ monomers (50/50 St/DVB) at $70{ }^{\circ} \mathrm{C}$ for $24 \mathrm{~h}$ : (A) $10 \mathrm{vol}$. \%, (B) $20 \mathrm{vol}$. \%, (C) 30 vol. \%, (D) 40 vol. \%, (E) 50 vol. \%, (F) 100 vol. \%.

SEM micrographs of poly(St-co-DVB) microspheres prepared in the mixture of acetonitrile and n-butanol are shown in Fig. 1(A-E). As seen in Fig. 1, very clear and smoothly shaped stable particles were obtained when less than 40 vol. $\%$ n-butanol was used. When up to $50 \mathrm{vol} . \%$ of $n$-butanol was added, the surfaces of the 
microspheres became rougher; some elliptic particles appeared. No stable microspheres were obtained when n-butanol was solely used without acetonitrile as seen in Fig. 1(F). n-Butanol was successful in the precipitation polymerization because it has similar solubility parameter to that of acetonitrile, $11.9\left(\mathrm{cal} / \mathrm{cm}^{3}\right)^{1 / 2}$ for acetonitrile and $11.4\left(\mathrm{cal} / \mathrm{cm}^{3}\right)^{1 / 2}$ for $\mathrm{n}$-butanol.

\section{Effect of Monomer Concentration}

Fig. 2 shows SEM photographs of microspheres prepared by precipitation polymerization of $50: 50$ vol. \% styrene and divinylbenzene with varying monomer concentration from 0.5 to 4 vol. \% relative to the reaction media with 20 vol. \% nbutanol used as cosolvent. A uniqueness of the precipitation polymerization compared with the conventional heterogeneous radical polymerization is the low monomer concentration [18]. In a monomer concentration range of $0.5-3 \mathrm{vol}$. \% relative to the reaction medium, stable microspheres without any coagulation were prepared, but partially coagulated particles were obtained up to 4 vol. $\%$ of the monomer concentration.

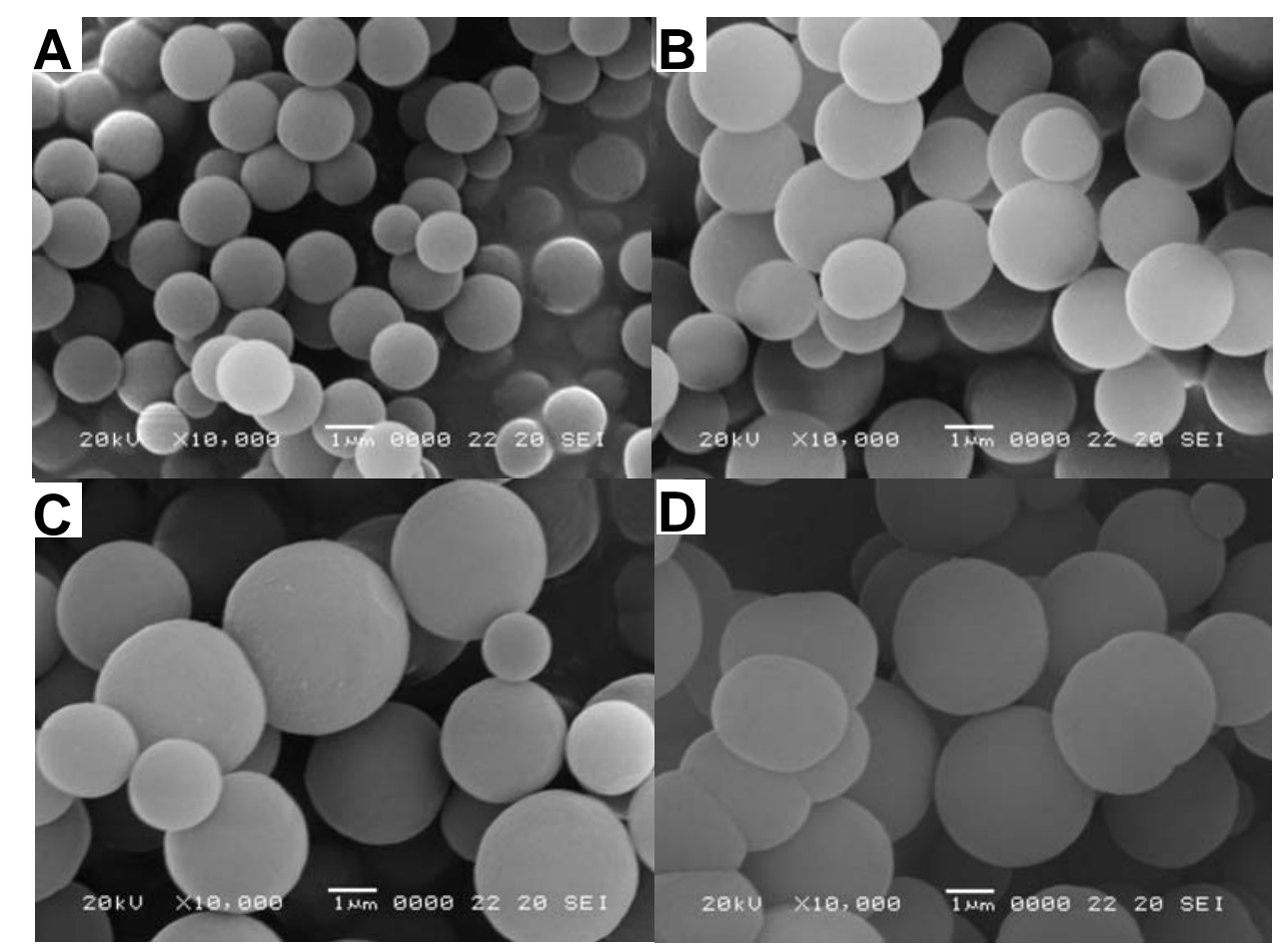

Fig. 2. SEM microphotographs of poly(St-co-DVB) particles prepared by precipitation polymerization composed of various concentration of monomers (50/50 St/DVB) with 1 wt. $\%$ AIBN in the mixture of 20 vol. $\%$ n-butanol and 80 vol. $\%$ acetonitrile at $70{ }^{\circ} \mathrm{C}$ for 24 h: (A) 0.5 vol. \%, (B) 1.5 vol. \%, (C) 3 vol. \%, (D) 4 vol. \%.

Fig. 3 depicts the change in the final particle size and uniformity $\left(D_{v} / D_{n}\right)$ with respect to the total monomers (St and DVB) concentration in the mixture of $80 \mathrm{vol}$. \% acetonitrile and 20 vol. \% n-butanol. The number-average diameters of the resulted particles increased significantly from $1.30 \mu \mathrm{m}$ for 0.5 vol. \% monomer loading to 2.27 $\mu \mathrm{m}$ for 3 vol.\% monomer loading. However, the uniformity remains intact with the variance in the monomer concentration. The increase in the size of the final microspheres is attributed to the change of the solvency of the medium. When the 
solubility parameter of the polymerization system becomes close to that of monomer, oligomeric species with a relatively high molecular weight are dissolved in the medium. Subsequently, the number of primary particles formed by precipitation of the oligomeric species is reduced and, finally, the particle size becomes larger. When the monomer concentration is increased, the solubility parameter of the reaction system approaches that of the monomer, which results in larger particles. The yield of the polymerization increased from $14 \%$ for 0.5 vol. \% of total monomer concentration to $48 \%$ for 5 vol. \%, which conformed to radical polymerization.

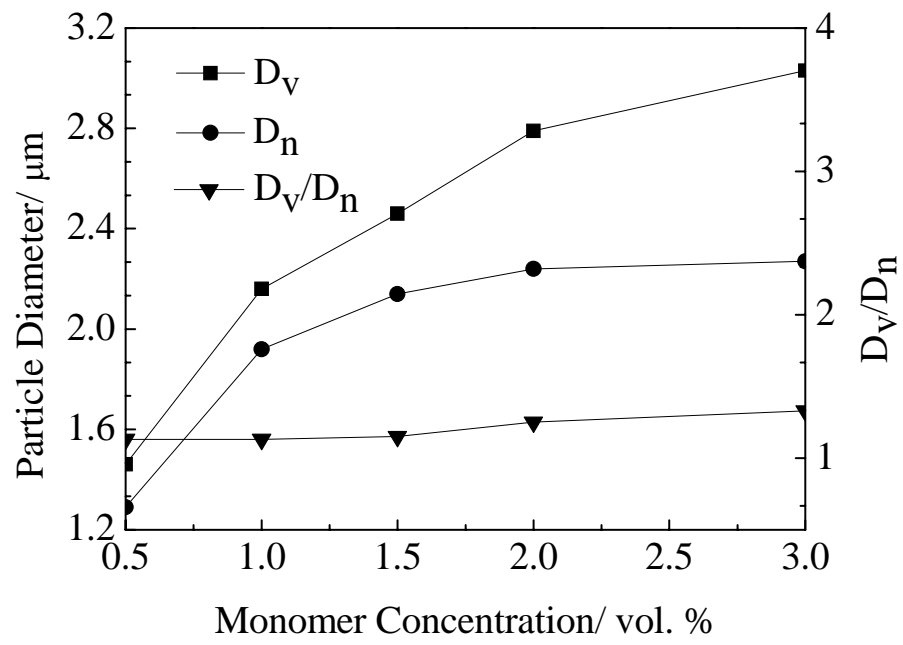

Fig. 3. Particle size and its uniformity of the microspheres prepared by precipitation polymerization of $1 \mathrm{wt}$. $\%$ AIBN with various concentrations of monomers at $70{ }^{\circ} \mathrm{C}$ for $24 \mathrm{~h}$.

\section{Effect of Initiator Concentration}

The particle size and uniformity $\left(D_{v} / D_{n}\right)$ of the resulted microspheres with respect to the concentration of initiator are shown in Fig. 4. The yield of polymerization with changing concentration of AIBN is depicted in Fig. 5. Both the size of the particles and the yield of the microspheres increased dramatically with increasing AIBN concentration. The $D_{n}$ of $1.35 \mu \mathrm{m}$ at $0.5 \mathrm{wt}$. \% AlBN increases to $2.59 \mu \mathrm{m}$ at $4.0 \mathrm{wt}$. $\%$ AlBN. Again, the uniformity is not significantly affected by the concentration of AIBN. The constant uniformity in precipitation polymerization would be owing to the different mechanism [19-21] of particle formation. At the initial stage of precipitation polymerization, the primary stable microspheres are generated by the aggregation of the primary nucleus particles. The nuclei formation is solely attributed to the crosslinked polymer molecules having a chain length greater than the critical limit which cannot be further dissolved in the reaction medium. Once the stable particles are generated by aggregation of the primary nucleus particles, the particles grow by continuously adsorbing the monomer or oligomeric species on the particle surface. Since no stabilizer used, the low molecular weight species are readily adsorbed onto the particle surface, which minimizes the formation of secondary particles. Thus, the uniformity would not be much influenced by monomer or initiator concentration. The yield of polymerization increased with the concentrations of AIBN. This may be because the higher initiator concentration would lead to the formation of precipitating large oligomeric species [12]. 


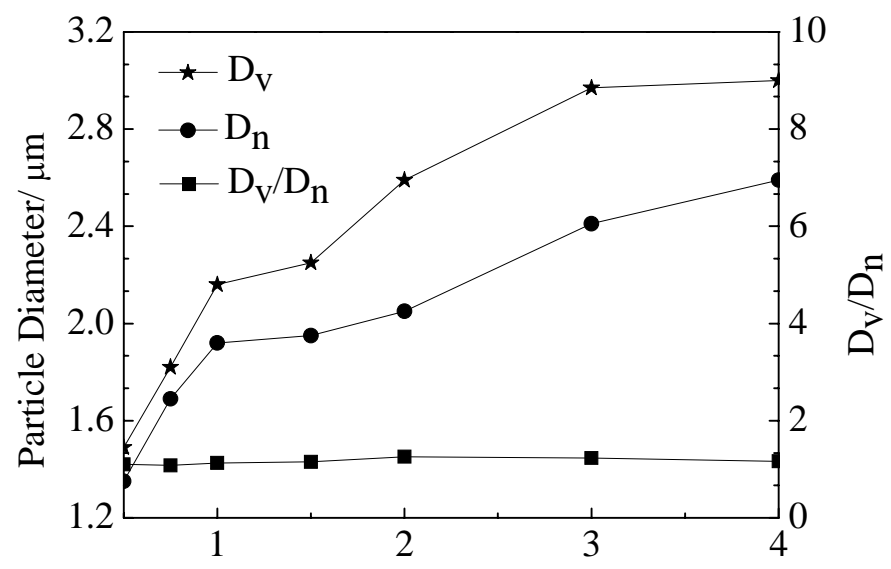

AIBN Concentration/ wt. \%

Fig. 4. Effect of the initiator concentration on the particle size and its uniformity of the microspheres prepared by precipitation polymerization with 1 vol. \% monomer in the mixture of 20 vol. \% n-butanol and 80 vol. \% acetonitrile at $70{ }^{0} \mathrm{C}$ for $24 \mathrm{~h}$.

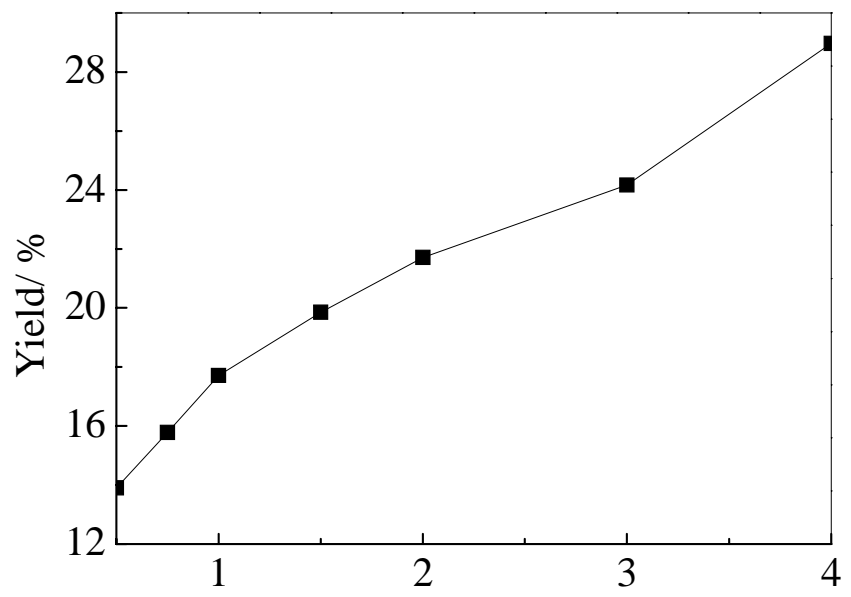

AIBN Concentration/ wt. \%

Fig. 5. Yield of polymerization with respect to the concentration of AIBN in the mixture of 20 vol. \% n-butanol and 80 vol. \% acetonitrile at $70{ }^{\circ} \mathrm{C}$ for $24 \mathrm{~h}$.

\section{Effect of DVB Concentration}

Fig. 6 represents the yield of the microspheres synthesized by precipitation polymerization in the mixtures of $20 \mathrm{vol}$. \% of n-butanol and $80 \mathrm{vol}$. \% of acetonitrile with 1 wt. \% AIBN and 1 vol .\% monomer at $70{ }^{\circ} \mathrm{C}$ for $24 \mathrm{~h}$, where the DVB concentration ranges form 20 to 90 vol. \% relative to the total monomers loading. As the DVB content increased form 20 to 90 vol. \%, the yield increased from $7 \%$ to 25 $\%$, respectively. The more the DVB content, the higher the yield obtained due to high degree of crosslinking [22]. No particles were obtained when DVB was not used. In precipitation polymerization, the presence of a crosslinkable monomer such as DVB is an essential feature. The formation of stable microspheres by precipitation polymerization is strongly dependent on the degree of crosslinking of the particles. 


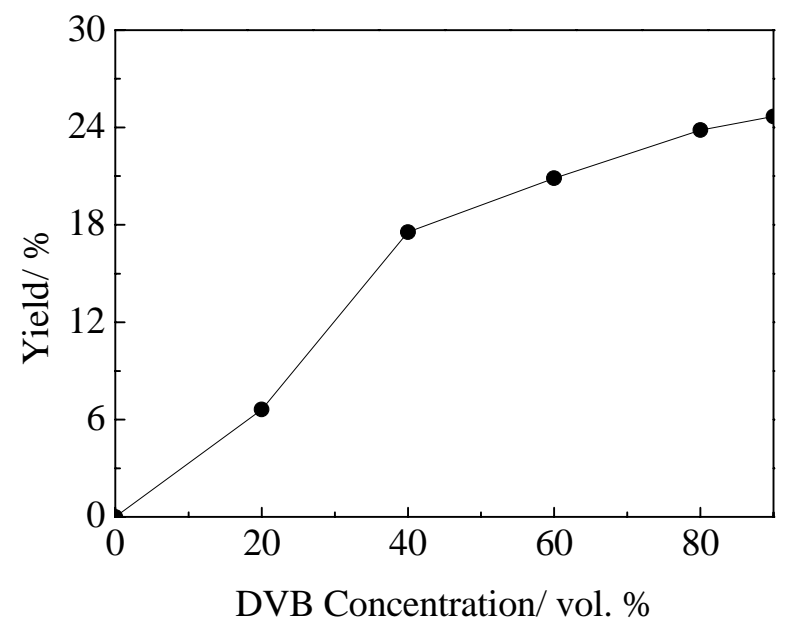

Fig. 6. Yield of precipitation copolymerization of St and DVB prepared with various concentration of DVB in the mixture of $20 \mathrm{vol}$. \% n-butanol and $80 \mathrm{vol}$. \% acetonitrile at $70^{\circ} \mathrm{C}$ for $24 \mathrm{~h}$.

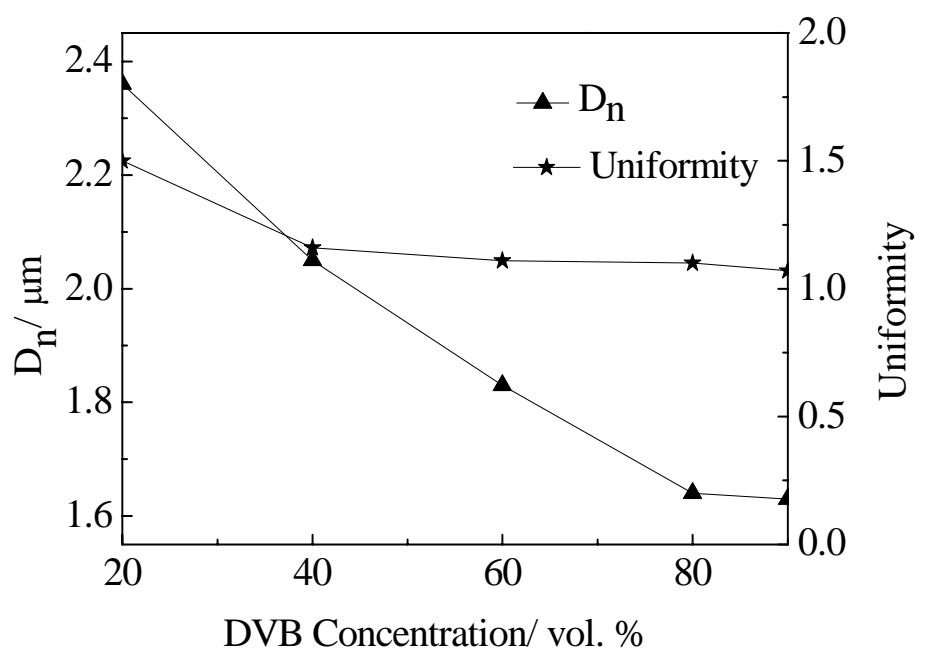

Fig. 7. Number-average particle size and the uniformity of poly(St-co-DVB) microspheres prepared with varying concentration of DVB in the mixture of $20 \mathrm{vol}$. \% n-butanol and 80 vol. \% acetonitrile at $70{ }^{\circ} \mathrm{C}$ for $24 \mathrm{~h}$.

In the course of the precipitation polymerization, the high degree of crosslinking within the polymer particles prepared prevents coagulation between the particles because of the improved hardness and the solvent-swollen gel layer on the surfaces.

Fig. 7 depicts the number-average particle size and the uniformity of the particles prepared using different concentration of DVB. The $D_{n}$ decreased from 2.36 to 1.63 $\mu \mathrm{m}$ with the concentration of DVB increasing from 20 to 90 vol. \%. In addition, the uniformity was reduced with the increasing concentration of DVB. The final particle size was determined from the initial number of nuclei that grow to become individual particles at the end of polymerization. If the small nuclei could be dissolved in the medium, the number of nuclei reduced. A small number of nuclei in the initial stage of polymerization lead to a larger particle size after completion of the polymerization. 

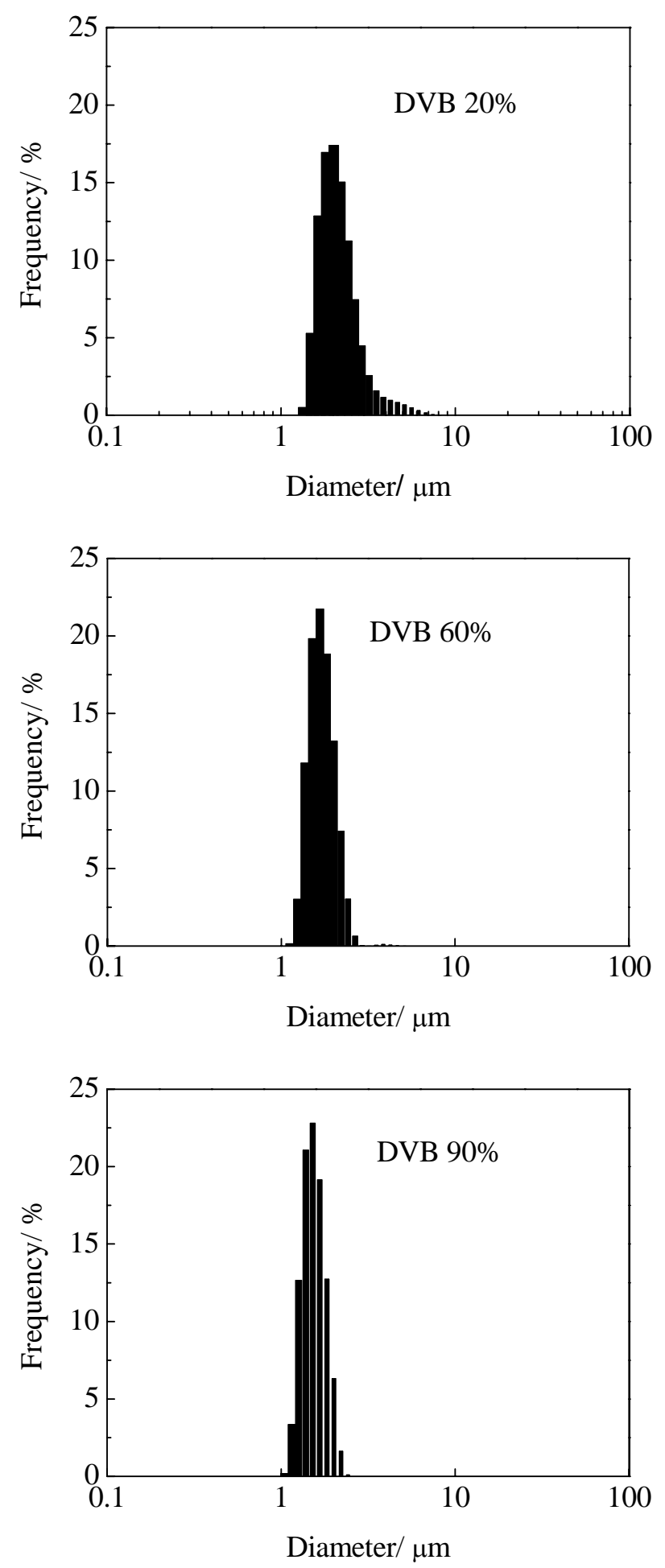

Fig. 8. Particle size distribution of poly(St-co-DVB) microspheres prepared by precipitation polymerization with $1 \mathrm{vol}$. \% monomer and $1 \mathrm{wt} . \%$ AIBN in the mixture of 20 vol. $\%$ n-butanol and 80 vol. $\%$ acetonitrile at $70{ }^{\circ} \mathrm{C}$ for $24 \mathrm{~h}$.

In this precipitation polymerization, n-butanol and acetonitrile used as the medium seems to be better solvent for St than DVB. As the concentration of DVB increases, the solubility of the nuclei in the medium would become poor, and then the final 
particle size would become smaller because of the larger number of nuclei formed in the initial stage [23].

The particle size distribution of poly(St-co-DVB) microspheres containing 20, 60, 90 vol. \% DVB are shown in Fig. 8. As mentioned in Fig. 7 the uniformity is enhanced as the concentration of DVB increases. In addition, no significant microsphere coagulation is observed in Fig. 8.

\section{Thermal Properties of Microspheres}

The thermal properties of the poly(St-co-DVB) microspheres obtained by the precipitation polymerization were characterized with DSC and TGA. The DSC thermograms of the poly(St-co-DVB) microspheres prepared with various concentrations of DVB from 20 to 90 vol. \% in the mixture of 20 vol. \% n-butanol and 80 vol. \% acetonitrile at $70{ }^{\circ} \mathrm{C}$ for $24 \mathrm{~h}$ are demonstrated in Fig. 9.

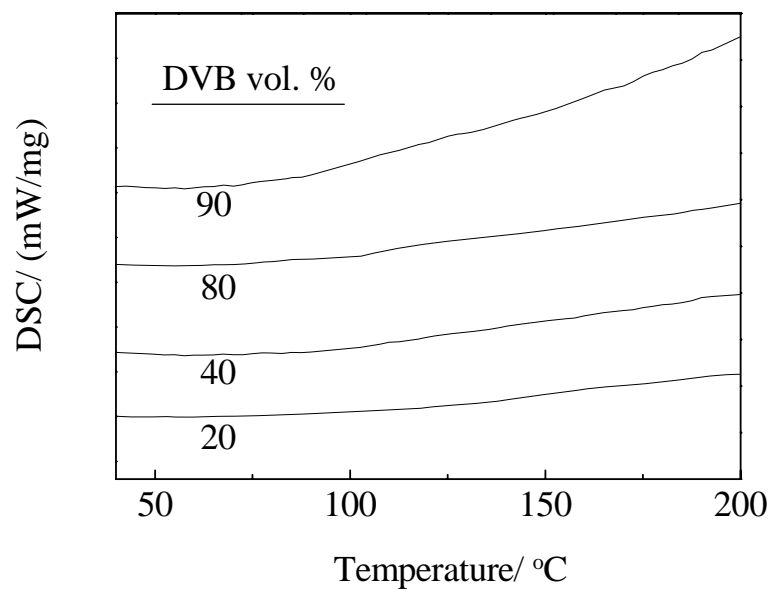

Fig. 9. DSC thermograms of poly(St-co-DVB) prepared with various concentrations of DVB in the mixture of 20 vol. \% n-butanol and 80 vol. $\%$ acetonitrile at $70{ }^{\circ} \mathrm{C}$ for $24 \mathrm{~h}$.

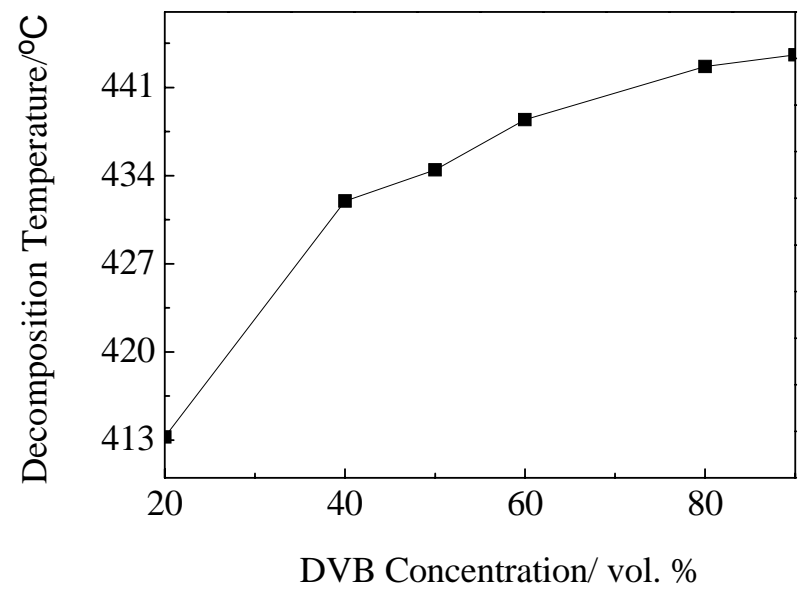

Fig. 10. Thermal stability of poly(St-co-DVB) microspheres containing an increasing concentration of DVB prepared by precipitation polymerization in the mixture of 20 vol. \% n-butanol and 80 vol. \% acetonitrile at $70{ }^{\circ} \mathrm{C}$ for $24 \mathrm{~h}$. 
A broadening and an increase in the endothermic transition followed by the disappearance of the glass transition temperature with increasing concentration of DVB were initially expected. However, no existence of the endothermic transition representing the glass transition temperature was observed for all examined samples. This may indicate that the whole poly(St-co-DVB) particles would be highly crosslinked [24].

Fig. 10 represents the decomposition temperature of poly(St-co-DVB) microspheres prepared by various polymerization techniques. In the case of the precipitation polymerization, the decomposition temperature varied from $413.24{ }^{\circ} \mathrm{C}$ to $443.59{ }^{\circ} \mathrm{C}$ for 20 and 90 vol. \% DVB, respectively. This indicates that the higher content of DVB improves the thermal stability of poly(St-co-DVB) particles due to increased degree of crosslinking between Sts [14].

\section{Conclusions}

Highly crosslinked, monodisperse poly(St-co-DVB) microspheres were prepared by precipitation polymerization in the mixture of acetonitrile and n-butanol. SEM characterization showed that the resulting microspheres had spherical shapes and a smooth surface. The particle size and yield increased with the concentration of monomer and initiator, while the uniformity was slightly influenced. $D_{n}$ decreased from 2.36 to $1.63 \mu \mathrm{m}$ with DVB concentration in monomer composition. In addition, the uniformity was reduced with the increasing concentration of DVB. In the measurements of the endothermic transition using DSC, no glass-transition temperature was observed for the whole samples synthesized in this study, implying that all microspheres are highly crosslinked. The decomposition temperature increased with the concentration of DVB and the thermal stability was significantly improved.

\section{Experimental part}

\section{Materials}

Styrene (St, A.R. grade, Tianjin Ruijinte Chemical Reagents Co., Tianjin, China) was purified by vacuum distillation before polymerization. Divinylbenzene (DVB, mixture of isomers: $\sim 80 \%$, Sigma-Aldrich, US) was used as received. 2,2'-Azobisisobutyronitrile (AIBN, A.R. grade, Tianjin Fuchen Chemical Reagents Co., Tianjin, China) was recrystallized from ethanol. Acetonitrile (A.R. grade, Tianjin Ruijinte Chemical Reagents Co., Tianjin, China) was used as solvent and used as received. n-Butanol (A.R. grade, Tianjin Ruijinte Chemical Reagents Co., Tianjin, China) was used as cosolvent and used as received. Ethanol (A.R. grade, Tianjin Ruijinte Chemical Reagents Co., Tianjin, China) was used without further purification.

\section{Polymerization}

The polymerization ingredients simply consisted of medium- various compositions of St and DVB comonomer mixture, and AIBN. The total amount of monomers was varied from 0.5 to $4 \mathrm{vol}$. \% of the medium. The concentration of AIBN was increased from 0.5 to $4 \mathrm{wt}$. \% with respect to the total amount of monomers used. After charging $50 \mathrm{ml}$ of the polymerizing mixture into a conical flask, it was purged with nitrogen for 15 min and sealed. Polymerization was carried out in a shaking water bath working with an agitation speed of $70 \mathrm{rpm}$ at $70{ }^{0} \mathrm{C}$ for $24 \mathrm{~h}$. After completion of 
the polymerization, the result particles were separated from the reaction medium by centrifugation and washed with ethanol repeatedly.

\section{Characterization}

The morphology of the poly(St-co-DVB) particles was determined by scanning electron microscope (SEM, JSM5610LV, JEOL, Japan), and the samples were sputter-coated with Au film before the examination.

The particle size and particle size distribution were determined by laser diffraction particle size analyzer (LS13320, Beckman Coulter, USA). The samples were dispersed in ethanol under ultrasonic vibrations before measurement.

Uniformity of the microspheres was calculated by the following formula:

$$
U=D_{v} / D_{n}
$$

where $D_{v}$ is volume-average diameter; $D_{n}$ is number-average diameter.

Differential scanning calorimetry (DSC, DSC204F1 Phoenix, Netzsch, German) was used to observe the glass-transition temperature of the microspheres. Thermal degradation of the crosslinked microspheres was studied using a thermogravimetric analyzer (TGA, STA409PC Luxx, Netzsch, German).

The yield of the polymerization was determined gravimetrically.

\section{Acknowledgements}

The authors are grateful for the financial support by the Natural Science Foundation of Shandong province (No. Q2006F05), the Applied Project of Educational Bureau of Shandong province (No. J08LC03).

\section{References}

[1] Urban, D.; Takamura, K. Wiley-VCH: Weinheim, 2002.

[2] Fudouz, H.; Xia, Y. Adv. Mater. 2003, 15, 892.

[3] Ugelstad, J.; Stenstad, P.; Kilaas, L.; Prestvik, W. S.; Rian, A.; Nustad, K.; Herje, R.; Berge, A. Macromol. Symp. 1996, 101, 491.

[4] Covolan, V. L.; Mei, L. H. I.; Rossi, C. L. Polym. Advan. Technol. 1997, 8, 44.

[5] Park, J. G.; Kim, J. W.; Oh, S. G.; Suh, K. D. J. Appl. Polym. Sci. 2003, 87, 420.

[6] Kiatkamjornwong, S.; Chientachakul, P.; Prasassarakich, P.; Damronglerd, S. J. Appl. Polym. Sci. 2001, 82, 1521.

[7] Liang, Y. C.; Svec, F.; Frechet, J. M. J. J. Polym. Sci., Part A: Polym. Chem. 1997, 35, 2631.

[8] Li, K.; Stöver, H. D. H. J. Polym. Sci., Part A: Polym. Chem. 1993, 31, 3257.

[9] Naka, Y.; Yamamoto, Y. J. Polym. Sci., Part A: Polym. Chem. 1992, 30, 1287.

[10] Frank, R. S.; Downey, J, S.; Stöver, H. D. H. J. Polym. Sci., Part A: Polym. Chem. 1998, 36, 2223.

[11] Li, W. H.; Stöver, H. D. H. J. Polym. Sci., Part A: Polym. Chem. 1999, 37, 2899.

[12] Yang, S.; Shim, S. E.; Choe, S. J. Polym. Sci., Part A: Polym. Chem. 2005, 43, 1309.

[13] Li, W. H.; Li, K.; Stöver, H. D. H. J. Polym. Sci., Part A: Polym. Chem. 1999, 37, 2295.

[14] Jin, J. M.; Lee, J. M.; Ha, M. H.; Lee, K.; Choe, S. Polymer, 2007, 48, 3107.

[15] Jin, J. M.; Yang, S.; Shim, S. E.; Choe, S. J. Polym. Sci., Part A: Polym. Chem. 
2005, 43, 5343.

[16] Shim, S. E.; Yang, S.; Jin, M. J.; Chang, Y. H.; Choe, S. Colloid. Polym. Sci. 2004, 283, 41.

[17] Bai, F.; Yang, X. L.; Huang, W. Q.; Macromolecules, 2004, 37, 9746.

[18] Goh, E. C. C.; Stöver, H. D. H. Macromolecules, 2002. 35, 9983.

[19] Shim, S. E.; Yang, S.; Choe, S. J. Polym. Sci., Part A: Polym. Chem. 2004, 42, 3967.

[20] Downey, J. S.; Frank, R. S.; Li, W. H.; Stöver, H. D. H. Macromolecules, 1999, $32,2838$.

[21] Downey, J. S.; Mclsaac, G.; Frank, R. S.; Stöver, H. D. H. Macromolecules, 2001, 34, 1543.

[22] Jin, J. M.; Lee, J. M.; Ha, M. H.; Lee, K.; Choe, S. Polymer, 2007, 48, 3107.

[23] Shim, S. E.; Yang, S.; Choi, H. H.; Choe, S. J. Polym. Sci., Part A: Polym. Chem. 2004, 42, 835.

[24] Ha, M.; Lee, K.; Choe, S. Polymer, 2008, 49, 4592. 